\title{
SHELAGH DELANEY Y EL DRAMA DE LA POSGUERRA: ROMPIENDO LOS CÁNONES ESTABLECIDOS
}

\author{
SHELAGH DELANEY AND THE DRAMA OF POSTWAR PERIOD: BREAKING \\ THE ESTABLISHED CANONS
}

Nerea Artesero-Bernal

Universidad de Sevilla

\section{RESUMEN:}

Este artículo trata de enfatizar el papel de la mujer en las artes escénicas. Resaltamos las obras de Shelagh Delaney (1939-2011); dramaturga británica de los años 50 . Nuestro objetivo es rescatar sus obras perdidas en el pasado; su primera creación teatral A Taste of Honey en el Theatre Royal Stratford East, en Londres, el día 27 de mayo de 1958. En conclusión, la obra que probablemente fue la más representada en la época de la posguerra (Dennis Barker).

\section{Palabras claves:}

Posguerra, el drama de la cocina, Jóvenes airados, Shelagh Delaney.

\section{Abstract:}

This article is based on the emphasis of women role in arts. We would like to concentrate on Shelagh Delaney's works (1939-2011); a British playwright in the 50s. Our aim is to rescue her lost plays from the past; her first creation was A Taste of Honey performed in the Theatre Royal Stratford East, in London, the 27th of May in 1958. In conclusion, probably the most performed work in the postwar era (Dennis Barker).

\section{KEY WORD:}

Postwar, Kitchen Sink drama, Angry Young Men, Shelagh Delaney. 


\section{THE KITCHEN SINK DRAMA Y LA INNOVACIÓN EN EL TEATRO}

A mediados y finales de 1950, se popularizó en Gran Bretaña este término que especifica un estilo de drama perteneciente a la posguerra que representaba los aspectos sórdidos de la realidad doméstica. Normalmente se utilizaba peyorativamente aplicado a obras que describían de una manera realista la vida de las clases trabajadoras de la época.

A este estilo se vinculan obras muy conocidas para la crítica como por ejemplo: Look Back in Anger de John Osborne de 1956 o en 1958 la obra de objeto de estudio de este artículo A Taste of Honey de Shelagh Delaney, entre otras... En este formato teatral, la gente vivía sus vidas en el escenario y para la mayoría de la audiencia es reflejo de sus propias vidas en el ámbito privado por lo tanto es una herramienta de hacer el teatro más accessible, a diferencia de la dramaturgia en el pasado, principalmente destinado a la élite de la sociedad burguesa.

Estas obras se centraban, metafóricamente, psicológicamente o en ciertas ocasiones literalmente en escenarios propios de las clases obreras y más concretamente en la cocina que ejercía diversas funciones: habitación, sala de estar y cocina. La cocina se convierte en un microcosmos en el que se descubren las relaciones personales y las carencias del mundo exterior (Susana Nicolás Román) y de ahí proviene el nombre de este género $(k i t c h e n=$ cocina)

Esta nueva tipología de obras implicaba un cambio trascendental en la manera de entender el teatro hasta el momento porque, a partir de 1956, el teatro inglés pasó a convertirse en noticia de primer orden. Fueron unos años esperanzadores, de cambio y renovación, en los que una pléyade de nuevos dramaturgos consiguió promover tal entusiasmo por el fenómeno teatral como no se conocía en Inglaterra desde la época de Bernard Shaw. Para Shaw, el teatro era en principalmente plantear un problema y hacer que los personajes argumentasen sobre él. Shaw inculcó a Inglaterra esta afición por las discusiones teatrales hasta el punto de que su influencia se muestra a través de la creación de obras futuras.

Un aspecto relevanteen estas obras del kitchen sink drama es que las artistas simpatizan hasta el extremo de convertir a los protagonistas en portavoces de sus propias inquietudes por lo que los personajes centrales transmiten sus propios sentimientos. La protagonista se caracteriza por la actitud crítica que mantiene frente a los demás personajes y frente a la realidad social de Inglaterra. En realidad, lo fundamental en el escenario es presentar ideas al público para que los espectadores, al retirarse a sus casas, reflexionasen solos o discutiesen unos con otros sobre lo que acabasen de escuchar. De hecho, se llegaba al punto de que era realmente de rigor, en una buena obra de teatro, atacar a la sociedad británica para poder conseguir la aprobación y los aplausos del público inglés.

La descripción de los personajes en las piezas de esta época son unos grandes bebedores, totalmente asqueados del mundo en que viven. El horror a todo es el tono de la juventud como ocurre en $A$ Taste of Honey donde la chica desgraciada da a luz un niño negro; tales son los asuntos que se incluyen en el teatro experimental llamado el Theatre Royal de Stratford, de Londres.

\section{Pinceladas biográficas de Shelagh Delaney}

Shelagh Delaney nació en el seno de una familia humilde el 25 de noviembre de 1938 en una ciudad industrial del norte llamada Salford, cerca de Manchester. Su padre era inspector de autobuses, un lector y un entusiasta cuentacuentos. Él le contaría con mucho talento sus experiencias en los Lancashire Fusiliers en el Norte de África. Tenía abuelos irlandeses, uno de ellos un apasionado socialista. Cuando tenía 12 años, se percató de que sabía escribir mejor que sus compañeros de clase. Su interés por el teatro aumentó a la vez que su interés por la escuela se desvanecía.

La aspirante a escritora tenía solo 19 años cuando escribió la obra de teatro A Taste of Honey en un período de 10 días basada en la relación entre una joven embarazada y un artista homosexual y se estrenó el 27 de mayo en 1958 en el Theatre Workshop en Stratford East, en Londres, y fue un éxito rotundo en el West End un año después.

Delaney estaba orgullosa de $A$ Taste of Honey y disfrutó de los beneficios materiales que eso le proporcionó. Cuando vendió los derechos de la obra para realizar la película sería dirigida por Tony Richardson y la actriz principal la joven Rita Tushingham y ganó 4 premios Bafta incluyendo mejor guion y película británica. “La película representa hechos controvertidos y difíciles de tratar- la pobreza y matrimonios de diferentes razas- aspectos con los que todavía tenemos que lidiar en la actualidad" (Tom Mullen)

Con respecto a la fama, también le agradaba, por lo menos al principio por los beneficios económicos que le generó y los caprichos que tenía ahora a su alcance y podía comprar. Un hecho reseñable a destacar es que tuvo el valor de publicar en un libro de 1964 titulado Sweetly Sings the Donkey las cartas de odio que recibía porque le divertían. Cito textualmente:

¡Vaya deshonra no sólo en el nombre del TEATRO sino para el género femenino! Tú que llevas la ropa sucia y el pelo y la piel mugrienta. ¿No te avergüenzas de ti misma?... Por cierto ¿cómo están tus amigos teddy boy'? ¿Llevan ellos navajas? Sería bastante gracioso si una noche ellos te apuñalan, te podría enseñar a escoger mejor tus gustos y compañías (Rachel Cooke)

1 Jóvenes que en los 1950 y 1960 vestían ropa de tipo Eduardiano.

2 Las citas son fragmentos obtenidos de periódicos ingleses y la traducción es mía.

Revist Internacional de Culturas y Literaturas, octubre 2016 
Sin embargo, A Taste of Honey se convertiria en una cruz que tendría que soportar, como suele ocurrir con el triunfo prematuro. La siguiente obra de Delaney, A Lion in Love de 1960, no tuvo ni la mitad del éxito que su predecesora Littlewood la leyó, y pensó: "No había aprendido nada" (Rachel Cooke)

La dificultad de escribir una segunda obra ideal ejercía presión sobre ella, no sólo porque su primera obra fuera exitosa debido a su aparente espontaneidad natural. Decepcionó las inmensas expectativas puestas en ella. A críticos conservadores como WA Darlington no les agradó este retrato de otra familia. No obstante, una nueva estirpe de críticos representados por Bernard Levin tenía una actitud más alentadora. Levin escribió: "El hecho de que, la señora Delaney no es sólo una astuta y una observadora perspicaz; ella es una artista muy delicada" (Dennis Barker)

Gradualmente comenzó a concentrarse en películas y la televisión en vez de seguir en escena, una transición que comentaba que estaba bien cuando funcionaba- pero normalmente no lo conseguía. Su posterior carrera como escritora de guiones en un primer momento intermitente, y después extinguida completamente: sus mayores éxitos fueron Charlie Bubbles, una película de 1967 en la que Albert Finney hace el papel de un exitoso pero frustrado escritor, y Dance With a Stranger, la película de Mike Newell de 1985 sobre Ruth Ellis, la última mujer británica en ser ejecutada en la horca por matar a su marido.

La mayoría de los últimos trabajos de Delaney fueron escritos para la radio. No es de extrañar, entonces, de que rara vez permitía que los teatros llevaran a escena nuevas producciones de su primera obra; quería ser recordada por ser más que la autora de A Taste of Honey. "Ella continuaba diciendo no" dice su hija, Charlotte (Rachel Cooke)

A pesar del cambio radical que presentó su carrera artística y que muchos coinciden en la opinión de que su valía no fue reconocida como se merecía esta dramaturga, en 1985, Delaney fue nombrada miembro de la Real Sociedad de Literatura. Shelagh Delaney murió el 20 de noviembre de 2011 en Suffolk a consecuencia de un cáncer de mama y legó los derechos de sus obras a su hija Charlotte para permitirle que si ella lo creía conveniente permitiera a los teatros exponer su obra de nuevo.

\section{El ORIGEN Y FUENTE DE INSPIRACIÓN DE LA OBRA A TASTE OF HONEY}

En cuanto al origen de $A$ Taste of Honey, su obra más conocida, la catapultó al éxito y a ser el alma de lo que se denominó en esta época el kitchen sink movement en Gran Bretaña durante el período de renacimiento tras la dramática posguerra.

Shelagh Delaney se pasó enfurecida cuatro noches para escribir una obra, escrita en una máquina de escribir prestada el día antes de su primer viaje al teatro, que alberga un realismo social impresionante, auténtico y sórdido que se convirtió en uno de los trabajos más influyentes de su generación que difería del contenido tradicional existente en los teatros decorosos acerca de la buena vida de la clase privilegiada.

Su obra se representó en Stratford sólo tres semanas después de la producción de John Gielgud de la obra de Terence Rattigan Variation On A Theme en el West End. Delaney fue a ver la obra de Rattigan al Manchester Theatre y fue su fuente de inspiración para componer A Taste of Honey con el objetivo de demostrar que ella lo podía hacer mucho mejor y combatir la insensibilidad demostrada por Rattigan en lo que concierne a los homosexuales.

El impacto teatral de esta obra se debe al dinamismo en el escenario por parte de Littlewood y a la visión compasiva de Shelagh Delaney hacia las clases trabajadores y sus aprietos. Obra que redacta una niña de Lancashire que había abandonado la escuela con 16 años; la obra trata una resistencia directa a las convenciones sociales y sexuales, una actitud sin prejuicios con respecto al mestizaje racial, y su aceptación de las expectativas, para las mujeres solteras, de una vida sin dependencia a los hombres fueron tan innovadoras como dos años antes sucedió con Look Back in Anger de John Osborne.

Pero en A Taste of Honey no aparecía ni la rabia ni la rebeldía de las obras de Osborne. Dejando a un lado la connotación sexual, la pieza evocaba las relaciones humanas desde una perspectiva de calidez y sarcasmo proponiendo un contraste reconstituyente al pesimismo; la heroína parece aceptar la miseria de su alrededor: "Realmente vivo en la misma época que yo misma, ¿no es verdad?" (The Telegraph)

En lo que respecta a la influencia e inspiración que generó la creación de la dramaturga en artistas posteriores:

1) En 1986, Morrissey el líder de los Smiths dijo: “Nunca he ocultado que Shelagh Delaney es la culpable de al menos el 50\% del motivo de mi escritura". Morrissey tomó como fuente de inspiración A Taste Of Honey para la letra de la canción I Don't Owe You Anything. Para los fans de Los Smiths, Delaney no necesita presentación. La imagen de la autora fue portada del álbum recopilación de 1987, Louder Than Bombs. El historiador de música Paul Wild asegura que Morrisey mostraba un inmenso respeto por los kitchen sink dramas de la época e idealizaba a las estrellas asociadas a éstos viéndolas como heroínas (Tom Mullen)

2) Para el guionista de televisión Tony Warren fue también una gran influencia el trabajo de esta dramaturga y se basó en sus temas para su más conocida creación Coronation Street. La famosa telenovela se estrenó por primera vez en 1960, dos años después del impacto de $A$ Taste of Honey en los escenarios. 
"Pero ella debería estar con los grandes. Ella fue una de las dramaturgas con mayor influencia del siglo y su influencia continúa palpable actualmente" expresaba el Dr. Lee un escritor que trabaja en la Universidad de Salford (Tom Mullen)

Delaney es un icono generacional para la juventud que captó las acciones reivindicativas expuestas en sus obras.

\section{SiNOPSIS Y RECEPCIÓN DE A TASTE OF HONEY (UN SABOR A MIEL $\left.{ }^{3}\right)$}

Una de las obras que caracteriza los movimientos feministas y clases trabajadoras de 1950. Esta obra se articula en torno a una madre y al personaje central de la pieza, su hija adolescente Jo, de clase obrera cuyas dificultades económicas extremas se palpan en el ambiente del apartamento decrépito de Salford donde conviven. A la madre se la retrata como una mujer que mendiga, a través de su cuerpo, la atención de hombres con dinero para ascender en el escalafón social y vivir en mejores condiciones.

Jo se siente sola y busca cariño en los brazos equivocados; un marinero negro que después de intimar una noche, la abandona y ella tiene que ocuparse del niño que viene en camino y sobrellevar las críticas y los prejuicios que conllevará su nacimiento debido al racismo que impregna la sociedad. Otros personajes incluyen al amante ruin de la madre, así como un estudiante de arte amable y homosexual que entabla amistad con esta chica durante el embarazo hasta que la madre, celosa por la relación que Jo y este chico tienen y más pendiente por el qué dirán que por el cariño y la compañía que se dan mutuamente, se empeña en echarlo de casa insistentemente y sin tacto alguno en lo que se podría considerar un estallido de amor maternal reprimido o una demostración de celos.

En Salford, la ciudad originaria de Delaney, el ayuntamiento se enfureció ya que el retrato de la ciudad les parecía un insulto- pero, cuando se convirtió en éxito desmedido y la autora en una celebridad nacional, pidieron una copia de su manuscrito para la biblioteca. Delaney la guerrera los denominó hipócritas y por este motivo le dio a Littlewood, directora del Theatre Workshop, el manuscrito original. A esta directora le debe en gran parte su éxito ya que amadrinó tanto la obra como a la dramaturga (Dennis Barker)

A Taste of Honey en un primer momento iba a ser una novela pero Delaney, como admitió después, estaría muy ocupada socializándose como para producir un libro de 80,000 palabras. La carrera que le siguió fue volátil y con altibajos. En 1960 A Taste of Honey se estrenó en Broadway (Nueva York) - con Joan Plowright interpretando a

3 El título con un toque dulce y edulcorado a priori nos deriva a pensar en un argumento dulce en un contexto de domesticidad y con personajes de la clase aristocrática. Sin embargo, es todo lo contrario y, de ahí, la ironía del elemento dulce de la miel en el título que se aleja mucho del argumento de la obra.
Jo y Angela Lansbury a su madre - y permaneció en cartelera durante casi un año. Plowright ganó un premio Tony gracias a su actuación.

El programa facilitado a la audiencia para $A$ Taste of Honey parece más un manifiesto político que teatral. Señalamos un fragmento: "proveniente de la ciudad Lancashire no devastada por la guerra sino por la industria y resiste al paro duradero, ella es la antítesis de los jóvenes airados londinenses. Ella conoce por qué está enfadada" (Rachel Cooke)

Se representa la obra con un estilo distintivo y semi-music hall ideado por la radical y polémica Joan Littlewood y la obra creó un revuelo con su honestidad estridente, integridad emocional, sarcasmo exuberante y toques de poesía. A Taste of Honey estuvo dos temporadas en el East End de Londres, en 1958 y en 1959, antes de que se transladó a Wyndham's para 350 representaciones. Se celebró como la personificación del movimiento de kitchen sink 10 años previos de la abolición de la censura. Mientras que ganó respeto crítico, el estilo del musical desenfadado de Littlewood no fue universalmente aceptado.

Cito literalmente las palabras de Lindsay Anderson de la revista Encore: "una trabajo de completa y estimulante originalidad. Un escape real del vacío conservador y burgués del West End". Para Alan Brien en The Spectator, aunque, no fue más que: "una anécdota trasnochada, exagerada de una obra que se desliza vacilante entre lo real y la fantasía, entre la farsa y la tragedia, entre lo afrodisíaco y lo vomitivo. Cada personaje aumenta su enfoque a través de diferentes lentes distorsionadas". La obra se escribe añadió Brien: "como si fuera un guion cinematográfico con un desprecio adolescente por lógica o forma o viabilidad sobre el escenario, y Miss Joan Littlewood la produjo con el tumultuoso resultado de un antiguo panfleto Living Newspaper" (The Telegraph)

A Shelagh Delaney se le consideraba la dramaturga más prometedora de su tiempo, una absoluta pionera pero un hecho a resaltar fue el misterio que se creó acerca de por qué nunca escribió nada tan importante como su primera obra. Según asegura el crítico John Russell Taylor: “a ciencia cierta ningún otro dramaturgo ha podido llegar tan lejos con un corpus de trabajo tan pequeño" (The Telegraph)

Es destacable cómo una adolescente y escritora emergente que vivía cerca de los muelles tuvo tanto éxito. El Dr Lee opinaba que el éxito de las obras de Delaney se ancla en la controversia: "hay que recordar que en esa época las obras estaban duramente censuradas. No se podía usar un lenguaje malsonante, ni retratar la homosexualidad ni otras muchas cosas que hoy en día damos por hecho" (Tom Mullen)

El Dr Lee creía que Delaney se las arregló para evadir a los censores a través de "la insinuación más que de la manifestación y exposición" - esta estrategia conllevó el disgusto de otros dramaturgos graduados universitarios predominantemente de 
clase media y tipos de Oxbridge (Oxford y Cambridge) quienes escribían obras nada exitosas de la época. "Y aquí estaba una colegiala que les estaba machacando a su propio juego". Consecuentemente, el Dr Lee expresa que el salto a la fama de Delaney se vio obstaculizado por las actitudes conservadoras de ese tiempo y ampliamente por una cuestión de su género. “Se cuentan historias sobre cómo intentó una vez abrir un museo de arte y la engañaron- simplemente porque ella era mujer y debería estar teniendo descendencia" (Tom Mullen)

Efectivamente, Delaney tuvo que sobrellevar duras críticas por su ataque a los convencionalismos del período. Su obra era innovadora con respecto a romper varios tabús discretamente observados en Noël Coward y Terence Rattigan, en cuyos teatros los personajes pertenecientes a las clases trabajadoras generalmente aparecían como alegres complementos y mayoritariamente presentaba a las mujeres como vírgenes o rameras. A Taste of Honey mostraba a las mujeres de clases trabajadoras desde una perspectiva de clase obrera, tenía un hombre homosexual como una figura central y solidaria, y un personaje negro que no era ni un estereotipo idealizado ni racial (Dennis Barker)

Incluso el actor que interpretó a Geoffrey, Murray Melvin, piensa que fue y aún sigue siendo ampliamente malentendida. "No había fregaderos aquí" (Rachel Cooke) La compañía the Roundabout theatre revivió la obra de $A$ Taste of Honey en Nueva York en 1981 con una nominación a premios Tony de Amanda Plummer en el papel de Jo.

\section{UNA ANGRY YOUNG MEN}

Los Angry Young Men (jóvenes airados) produjeron una auténtica convulsión en la tranquila literatura británica. La prensa inventó la etiqueta de los Angry Young Men como una estrategia de lanzar a la fama determinadas obras de teatro. Se trata de un nuevo grupo de artistas fehacientes defensores de las clases humildes y que luchan contra lujos y la alta burguesía.

El movimiento estético de los Angry Young Men simboliza una furiosa rebeldía y además demuestra una abierta reacción ante una inevitable crisis social combinada con voces idealistas: "porque sé que los sueños triunfarán" (Cándido Pérez Gallego) Desaparece en las obras todo lo que implica una innovación en el lenguaje o sentimentalismo. La estética de este movimiento tiende a configurar situaciones extremas y a despojar al ser humano de todo lo gratuito, hacia un camino que le lleve a vivir. Quieren ponderar la necesidad de una literatura sin tantas preocupaciones estilísticas y más interés social, como más efectiva en momentos determinados de la historia.
Entonces ¿en qué consiste el mérito de los jóvenes airados?: son héroes/heroínas que descubrían una realidad que había sido olvidada durante años por los "artistas oficiales". Su actitud ante la vida es de enfrentarse contra la adversidad por la ausencia de algo que considera básico y necesario.

Los protagonistas de clases humildes sienten las barreras de la sociedad con gran dureza por lo tanto intentan ascender de clase, "aburguesarse", lo cual esconde un trágico sentimiento de renuncia a lo que es su mundo natural. Plantean un deseo de abandonar la sociedad con una mezcla de anhelo de superación y egoísmo. La solución que se consigue en las obras es una amarga violencia en el lenguaje, un carácter inadaptado de los personajes y necesidad de cambio con el objetivo de mejorar y ser felices.

A través de las crónicas documentales reproducidas por los Angry Young Men podemos seguir acontecimientos que vemos todos los días en Inglaterra que no marcan una realidad para las clases pudientes alejadas obsesivamente de todo lo que significa una problemática social.

A Shelagh Delaney le acompañó la connotación de Angry Young Woman ya que ella conocía a la perfección por qué estaba enfadada. Hay que apostar que Delaney estaba harta del sistema de clases rígido de Gran Bretaña y especialmente del rol de las mujeres dentro de este sistema. Tenía muchísimas cosas que decir al respecto tanto en relación a la manera en que las reformas sociales no habían conseguido mejorar el grupo de los pobres y en lo que respecta a la sexualidad; se considera por lo general $A$ Taste of Honey como la primera obra moderna que describe a un hombre homosexual de clase trabajadora. Su trabajo es el polo opuesto a la educación y al decoro, características que se supone que las mujeres debían tener.

Pero no es correcto ver a la implacable Delaney como una estrella solitaria, como la única miembro femenina de la nueva ola que es como la mayoría de las veces se le retrata. Había otras mujeres enfadadas a su alrededor, chicas resueltas y cabezona como ella. Como por ejemplo, poco después de que la obra de Delaney se estrenara, el Royal Court apostó por el The Sport of My Mad Mother de Ann Jellicoe; presentan elementos de género en sus obras como parte de la crítica social hasta este momento ausente en los Angry Young Men puesto que dirigen su ira hacia cuestiones sociales y políticas pero no de género.

\section{Homenaje Para Shelagh Delaney}

El 17 de noviembre de 2014 tiene lugar una conmemoración de los fans de Shelagh Delaney en Saldford su ciudad natal para recordarla y se lleva a cabo un acto simbólico: rellenar botes con miel en honor al título de su popular obra A Taste of Honey. En esta 
fecha, ella celebraría su 76 cumpleaños. Los fans marcan un antes y un después porque se proclama el primero de muchos días de Shelagh Delaney.

A pesar de que Delaney deslumbró a Broadway con una obra que escribió en una edad muy temprana y posteriormente inspiraría en sus canciones a la leyenda del rock Morrisey. "Y conseguir todo esto en una edad tan prematura es reseñable. Ella es una autora que se mantiene vigente. Sin embargo estoy totalmente de acuerdo con la afirmación de que no ha recibido todo el reconocimiento merecido" (Tom Mullen).

Por este motivo, se produce un evento que tuvo lugar en el Teatro de Artes de Salford cuyo interés era una reconstrucción de una escena de la más famosa obra de Shelagh Delaney, A Taste of Honey.

Recientemente del 30 de enero al 6 de febrero de 2016 se ha homenajeado a la dramaturga mediante una visualización de la obra A Taste of Honey en el Stockport Garrick Theatre sabiamente anunciado mediante la red social Facebook para lograr una mayor difusión y expansión de Shelagh Delaney a la audiencia actual.

“¿Pero alguien sabe quién fue Delaney? ¿Cómo logró tanto éxito alguien de cuna humilde y que logre que su trabajo sea todavía hoy en día relevante?" (Tom Mullen)

Charlotte Delaney, su hija, después de la muerte de su madre decidió permitir que el National Theatre expusiera $A$ Taste of Honey y concluye que cree que la compañía teatral ha captado la esencia de la obra. Charlotte afirma que: "Para mí, es una obra sobre las mujeres-las madres y las hijas-y ellas han sido capaces de exteriorizarlo. Pero eso no quiere decir que a mi madre le hubiera parecido bien. Cuando se estaba muriendo, me dijo que estaba contenta de dejarlo atrás, este cuerpo de trabajo. Pero la verdad es que si ella estuviera viva, no habría aceptado su representación ni tan siquiera en el National Theatre. A ella no le gustaba la atención" (Rachel Cooke)

Le prosiguen preguntando en la entrevista los adjetivos que utilizaría para describir a Shelagh Delaney porque aparentemente sonaba muy cabezona y tremenda. Charlotte Delaney detalla que: "Era una buena amiga, lea, una abuela fabulosa. Ella siempre estaba bromeando - nunca perdió eso - y nunca cambió por la fama, ni incluso cuando Katharine Hepburn la llevó a dar una vuelta en el coche de Spencer Tracy. Hablaba con cualquiera sin importarle quién fuera: Ringo Starr o la madre de su vecina. Socialista, irritante, aventurera... y una renegada" (Rachel Cooke)

\section{CONCLUSIONES}

Hoy nadie parece dudar que el texto de una obra sólo existe en virtud de su realización sobre un escenario, puesto que el teatro no se escribe para ser leído, sino para ser representado. El teatro es y será siempre la más viva de las artes, la más inmediata, mucho más que el cine, que es la imagen de lo que ha sido representado antes.

Shelagh Delaney era especial, o inmensamente talentosa. Pero presentar a una mujer brillante; se insinúa que cualquier éxito que ella disfrutó fue accidental - y no hubo ningún accidente aquí. En Stratford, hay una copia de la carta extraordinaria que Delaney escribió a Littlewood cuando le mandó $A$ Taste of Honey, y justamente quita la respiración. Las agallas, el espíritu. Disculpándose por su escritura, Delaney le dice a Littlewood que: "si ella puede encontrar algún sentido de su obra de teatro (o incluso un pequeño sin sentido) estaría agradecida de su crítica - aunque odia las críticas de este tipo". Y después, una última puñalada, modesta pero no, notarás, falsamente: "yo quiero escribir para el teatro, pero sé muy poco sobre él. No sé nada, no tengo nadaexcepto ganas de aprender - e inteligencia. Sinceramente tuya, Shelagh Delaney" (Rachel Cooke)

Delaney comparte con sus colegas varones de los Angry Young Men la crítica al Establishment, a la burguesía, a la política... Pero una innovación a tener en cuenta es que gracias a estas mujeres encontramos el aspecto de género como parte de esta ira ya que por parte de los hombres las mujeres quedaban silenciadas, minimizadas y por eso es importante dar visibilidad a las mujeres dramaturgas que sí están escribiendo en esta época y critican al patriarcado.

\section{REFERENCIAS BIBLIOGRÁFICAS}

Barker, D., Shelagh Delaney obituary. The Guardian. 21 November 2011. Internet. 11-04-16. <http://www.theguardian.com/stage/2011/nov/21/shelagh-delaney> Cooke, R., Shelagh Delaney: the return of Britain's angry young woman. The Observer. 27 January 2016. Internet. 29-03-16.

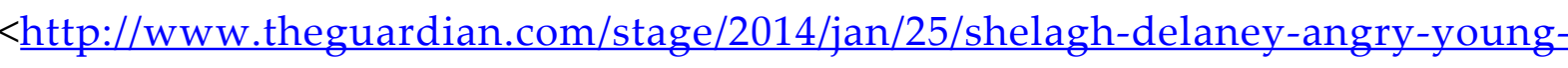
woman-a-taste-of-honey>

Mullen, T., Morrissey's idol: Celebrating Shelagh Delaney Day. BBC News. 25

November 2014. Internet. 21-03-16. <http://www.bbc.com/news/uk-englandmanchester-30148668>

Nicolás Román, S., “Roots y Serjeant Musgrave's Dance”, Director de tesis Dr. Juan José Torres Núñez, Voces femeninas en el teatro de Edward Bond, Universidad de Almería, Departamento de Filología Inglesa y Alemana, 2007, pp.155.

Osborne, J., Looking Back. Never Explain, Never Apologise, Faber\&Faber Limited, London, 1999. 
Pérez Gallego, C. Literatura y rebeldía en la Inglaterra actual: los Angry Young Men, un movimiento social de los años cincuenta, CSIC, Madrid, 1968, pp.209, 211.

Portillo García, R., El autor a la búsqueda de un protagonista: las obras dramáticas de John Osborne, 1956-1966, Tesis Doctoral, Universidad de Salamanca, Salamanca, 1978.

Shelagh Delaney. The Telegraph. 21 November 2011. Internet. 10-04-16.

http://www.telegraph.co.uk/news/obituaries/culture-obituaries/theatre- obituaries/8905444/ Shelagh-Delaney.html 